\title{
Assessing and mitigating alignment defects of the pyramid wavefront sensor: a translation insensitive control method (Corrigendum)
}

\author{
V. Deo ${ }^{1}$, É. Gendron ${ }^{1}$, G. Rousset ${ }^{1}$, F. Vidal ${ }^{1}$, T. Buey ${ }^{1}$, and M. Cohen ${ }^{2}$ \\ ${ }^{1}$ LESIA, Observatoire de Paris, Université PSL, CNRS, Sorbonne Université, Univ. Paris Diderot, Sorbonne Paris Cité, \\ 5 place Jules Janssen, 92195 Meudon, France \\ e-mail: vincent. deo@obspm. fr \\ 2 GEPI, Observatoire de Paris, Université PSL, CNRS, 5 place Jules Janssen, 92195 Meudon, France
}

A\&A 619, A56 (2018), https: //doi .org/10 . 1051/0004-6361/201833685

Key words. instrumentation: adaptive optics - techniques: high angular resolution - telescopes - errata, addenda

\begin{abstract}
An error has occurred during the production of the published article. Before Eq. (4), second column, fourth line, it is written: "Normalized quadrants, either local or global, are", but it should read "Normalized quadrants, either local or global, are rearranged into measurement terms depending on the PWFS operation method. Conventionally, quad-cell SH-like terms X, Y are used as follows:"
\end{abstract}

$$
\begin{aligned}
{\left[\begin{array}{c}
X_{\text {norm }} \\
Y_{\text {norm }}
\end{array}\right][k] } & =\left[\begin{array}{l}
A_{\text {norm }}-B_{\text {norm }}+C_{\text {norm }}-D_{\text {norm }} \\
A_{\text {norm }}+B_{\text {norm }}-C_{\text {norm }}-D_{\text {norm }}
\end{array}\right][k] \\
& =\left[\begin{array}{cccc}
{[r] 1} & -1 & 1 & -1 \\
1 & 1 & -1 & -1
\end{array}\right] \cdot\left[\begin{array}{l}
A_{\text {norm }} \\
B_{\text {norm }} \\
C_{\text {norm }} \\
D_{\text {norm }}
\end{array}\right][k],
\end{aligned}
$$

where norm $\in\{$ Loc, Glob $\}$. 